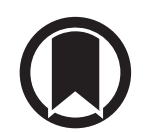

CrossMark

\section{Exercise pulmonary haemodynamic response predicts outcomes in fibrotic lung disease}

To the Editor:

Pulmonary hypertension is diagnosed by an elevated mean pulmonary arterial pressure (mPAP) during resting right heart catheterisation (RHC) [1]. Although "exercise-induced pulmonary hypertension" is no longer a distinct clinical entity, recently, there has been renewed interest in the prognostic value of pulmonary haemodynamic responses to exercise, particularly in patients with fibrotic lung disease [1-4]. As the optimal use of exercise RHC in this cohort remains unknown, our main objective in this study was to study the relationship between exercise haemodynamics and outcomes in fibrotic lung disease patients [3-7].

All consecutive fibrotic lung disease patients undergoing exercise RHC testing at our centre over a 7-year period, regardless of the MPAP at rest, were included in the study. Fibrotic lung disease, scored by one observer (A. Jose) using the method of $\mathrm{GoH}$ et al. [8], was defined as $>10 \%$ fibrosis on computed tomographic imaging at the time of exercise RHC. Subjects without fibrotic lung disease, those with missing data and those with an elevated pulmonary artery wedge pressure (PAWP) at rest ( $>15 \mathrm{mmHg}$ ) were excluded.

Measurements at rest included heart rate, right atrial pressure (RAP), mPAP, digital mean PAWP, thermodilution cardiac output (CO) and pulmonary artery oxygen saturation. Pressures were measured as the digital mean across several respiratory cycles. Pulmonary vascular resistance (PVR) was calculated in the standard fashion. After baseline haemodynamic measurements, the patients underwent supine bicycle ergometer exercise to (subjective) maximal exertion and haemodynamic measurements were repeated.

The total pulmonary resistance was calculated both at rest $(\mathrm{TPR}=\mathrm{mPAP} / \mathrm{CO})$ and at peak exercise (TPRex). The exercise-induced TPR $(\Delta \mathrm{TPR} e x=\Delta \mathrm{mPAP} / \Delta \mathrm{CO})$ was calculated from the difference between rest and peak exercise haemodynamics [1, 3, 5-7]. An abnormal precapillary $\Delta$ TPRex, reflecting pulmonary arterial response to exercise, was defined as $\Delta \mathrm{TPRex} \geqslant 3 \mathrm{mmHg} \cdot \mathrm{L}^{-1} \cdot \mathrm{min}^{-1}$ with concomitant PAWP $<20 \mathrm{mmHg}$ at peak exercise. This cut-off was used to definitively exclude patients with occult left heart disease $[1,7]$. Demographic, laboratory, functional testing (including 6-min walk test (6MWT)) and pulmonary function testing (PFT) were collected within 3 months of the index exercise RHC.

The primary outcome was defined as the first of three clinical worsening events: hospitalisation for cardiopulmonary decompensation, lung transplantation or death. Information on the primary outcome was obtained from the medical record and review of patient's charts. Time zero was the date of the index RHC, and patients were followed until either an event occurred or the study period ended. Patients lost to follow-up were censored at the time of their last clinical encounter. This study was reviewed and approved by the Inova Institutional Review Board (IRB 15-2025).

Differences in clinical and haemodynamic characteristics between groups were compared using the Wilcoxon rank-sum test for continuous variables and the Chi-squared test for categorical variables. A two-tailed p-value $<0.05$ was considered significant. Cox proportional hazard models were used to assess the relationship between variables and time to clinical worsening [9]. The variance inflation factor was used to test for collinearity, with variables being retained on the basis of their univariable $p$-value. Non-collinear individual variables with a $\mathrm{p}$-value $<0.1$ in the univariable analysis were incorporated into

@ERSpublications

Haemodynamic changes with exercise, including $\Delta \mathrm{mPAP} / \Delta \mathrm{CO}$, predict clinical worsening in fibrotic lung disease patients http://ow.ly/uKno30lb5fF

Cite this article as: Jose A, King CS, Shlobin OA, et al. Exercise pulmonary haemodynamic response predicts outcomes in fibrotic lung disease. Eur Respir J 2018; 52: 1801015 [https://doi.org/10.1183/ 13993003.01015-2018]. 
the final multivariable model, which was subsequently adjusted for age, sex, RAP, resting mPAP, resting $\mathrm{CO}$, baseline 6MWT and the presence of targeted pulmonary arterial hypertension (PAH) therapy. Additionally, an unadjusted multivariable analysis excluding patients with an exercise PAWP $\geqslant 20 \mathrm{mmHg}$ was performed to exclude those patients with occult post-capillary disease. This model was constructed using forwards and backwards selection, retaining variables based on the likelihood ratio test.

The proportional hazards assumption was examined for all models using scaled Schoenfeld residuals. All analyses were performed using R version 3.3.2 (R Foundation for Statistical Computing, Vienna, Austria).

TABLE 1 Baseline demographic, clinical and haemodynamic characteristics, and Cox proportional hazards models

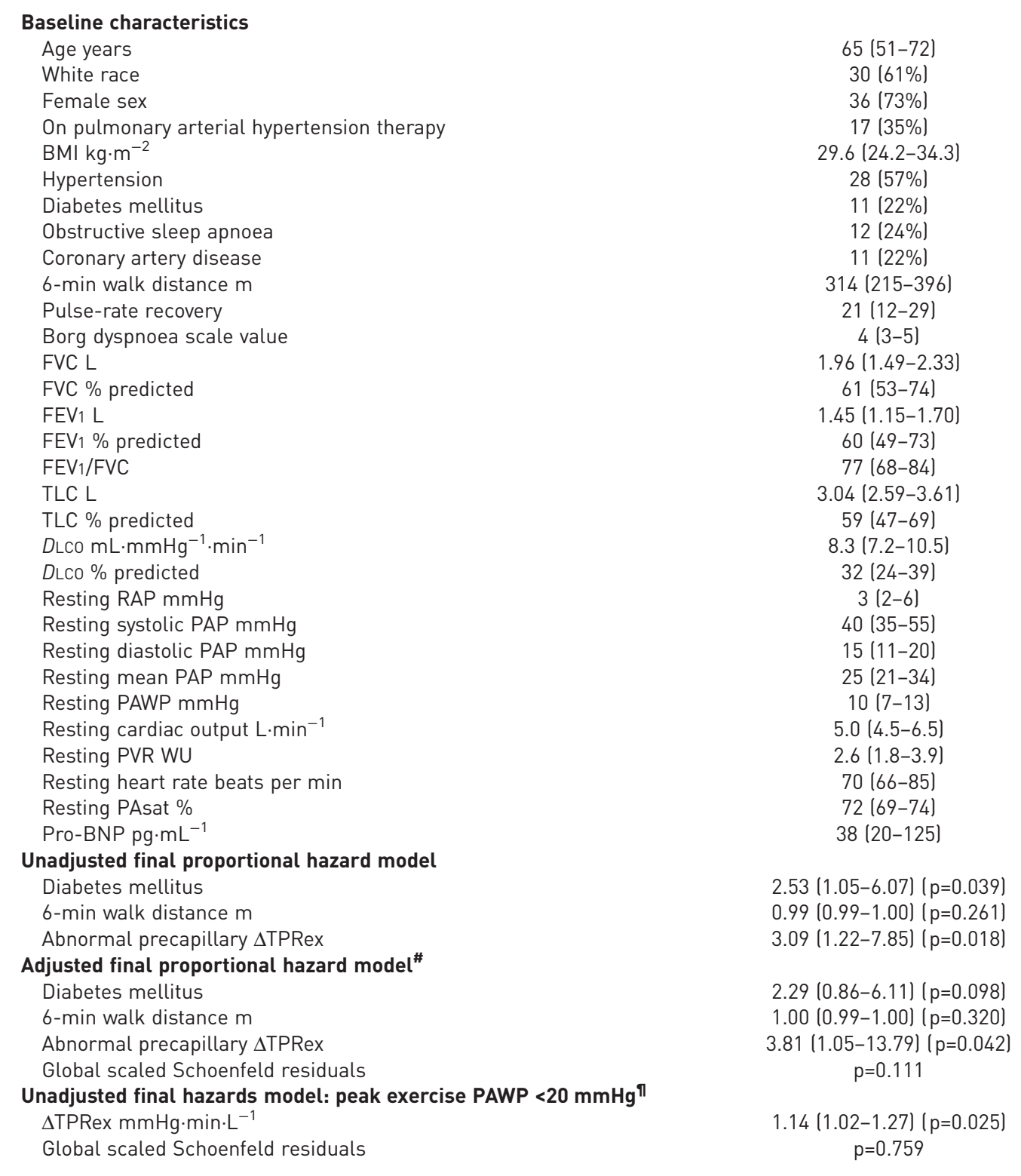

Data are presented as median (interquartile range) or hazard ratio $(95 \% \mathrm{CI}$ ), unless otherwise stated. BMI: body mass index; FVC: forced vital capacity; FEV1: forced expiratory volume in $1 \mathrm{~s}$; TLC: total lung capacity; $D\llcorner C 0$ : diffusing capacity of the lung for carbon monoxide; RAP: right atrial pressure; PAP: pulmonary arterial pressure; PAWP: pulmonary artery wedge pressure; PVR: pulmonary vascular resistance; WU: Wood unit; PAsat: pulmonary artery saturation; BNP: brain natriuretic peptide; $\triangle T P R e x$ : exercise induced change in total pulmonary resistance. \# : adjusted for age, sex, resting RAP, resting mean PAP, resting cardiac output and resting 6-min walk distance, and stratified on the presence of pulmonary arterial hypertension therapy; ${ }^{\text {ก: }} \mathrm{n}=22$. 
A total of 119 patients underwent exercise RHC, of whom 49 had fibrotic lung disease and were included in the final analysis. The most common aetiology of lung disease was connective tissue disease associated (39\%), followed by nonspecific interstitial pneumonitis (18\%), and combined pulmonary fibrosis and emphysema (14\%).

The baseline characteristics of patients are shown in table 1. Patients were predominantly older Caucasian females. As expected, all patients displayed physiological restriction and diffusion capacity impairment on PFT. A number of patients (55\%) had $\mathrm{PH}$ at rest and 35\% were on targeted PAH therapy at the time of the exercise RHC testing. The median observation period was 11 months and a total of 25 patients (51\%) experienced the primary outcome of clinical worsening. The most common primary outcome event was hospitalisation, the majority $(80 \%)$ driven by interstitial lung disease exacerbations. A total of four patients died during the study period and one patient underwent lung transplantation.

As compared to stable patients, those with clinical worsening had a significantly higher resting PVR, lower resting RAP and PAWP, and lower 6MWT distance. Additionally, the presence of diabetes mellitus was significantly more common. Although there were no significant differences in exercise haemodynamics, a greater proportion of patients with clinical worsening (40\% versus $17 \%$ ) had an abnormal pre-capillary $\triangle$ TPRex.

The final multivariable models are shown in table 1 . After adjustment, only an abnormal pre-capillary $\Delta$ TPRex was significantly predictive of clinical worsening (hazard ratio 3.81, $\mathrm{p}=0.042$ ). When limiting the analysis to patients with an exercise PAWP $<20 \mathrm{mmHg}$, the $\triangle \mathrm{TPRex}$ had the strongest association with clinical worsening.

Our study demonstrates that abnormal pulmonary exercise parameters, specifically an abnormal precapillary $\triangle \mathrm{TPRex}$, may predict clinical outcomes in patients with fibrotic lung disease. This variable may reflect the underlying pulmonary vascular abnormalities present in some patients with fibrotic lung disease [10-12]. Functionally, abnormal elevations in the $\triangle T P R e x$ with exercise may reflect an impaired relationship between $\mathrm{mPAP}$ and $\mathrm{CO}$ due to a limited pulmonary vascular reserve, recruitment and compliance. Independent of the pulmonary vasculature, these abnormalities may also be a consequence of hypoxic pulmonary vasoconstriction during exercise and fibrotic destruction of the pulmonary capillary bed $[5,6,10-13]$. Interestingly, our results suggest that the presence of diabetes mellitus might also be associated with worse outcomes. While our study was not designed to study this specific relationship, we suggest that the association between exercise haemodynamics, metabolic dysfunction, pulmonary vascular remodelling and clinical outcomes warrants further study [14].

In contrast to previous studies in patients with idiopathic pulmonary fibrosis, we found no association between the PFT measures of disease severity and outcomes [15]. This may be due to the small numbers and heterogeneous aetiologies of lung fibrosis in our study.

Our study has a number of strengths, including a well-described population of patients undergoing exercise RHC and accounting for known markers of clinical worsening in our final model. However, there are also some limitations. The study population included patients from a single tertiary referral centre, some also predisposed to PAH due to conditions like scleroderma, and thereby on targeted PAH therapy. This could potentially have introduced a certain selection bias and limits the generalisability of our findings.

In conclusion, exercise pulmonary haemodynamic response in the form of an abnormal pre-capillary $\Delta \mathrm{TPRex}$ is significantly and independently predictive of adverse clinical outcomes in patients with fibrotic lung disease. Utilisation of haemodynamic information obtained during exercise RHC testing might be a useful tool to risk-stratify patients with fibrotic lung disease. Further studies are encouraged to validate our findings.

Arun Jose $\oplus^{1}$, Christopher S. King ${ }^{2}$, Oksana A. Shlobin ${ }^{2}$, A. Whitney Brown ${ }^{2}$, Chengxi Wang ${ }^{3}$ and Steven D. Nathan ${ }^{2}$

${ }^{1}$ Pulmonary, Critical Care and Sleep Medicine Division, The George Washington University MFA, Washington, DC, USA. ${ }^{2}$ Lung Disease and Transplant Program, Inova Heart and Vascular Institute, Inova Fairfax Hospital, Falls Church, VA, USA. ${ }^{3}$ Internal Medicine Division, Inova Fairfax Hospital, Falls Church, VA, USA.

Correspondence: Arun Jose, Pulmonary, Critical Care and Sleep Medicine Division, University of Cincinnati, Medical Sciences Building Suite 6352-A, 231 Albert Sabin Way, Cincinnati, OH 45229, USA. E-mail: josean@ucmail.uc.edu

Received: April 232018 | Accepted after revision: July 252018

Conflict of interest: C.S. King reports serving on an advisory board for Boehringer Ingelheim outside the submitted work. A.W. Brown reports serving on an advisory board in July 2017 for Promedior, serving on an advisory board in October 2017 for and receiving speakers' bureau training in April 2018 from Genentech, and serving as a consultant on curriculum development for a continuing medical education (CME) programme and speaking at CME presentations at medical grand round venues in the community for PILOT for IPF, outside the submitted work. S.D. Nathan has been a consultant for Bayer Pharmaceuticals, Bellephoron, United Therapeutics, Actelion and Third Pole in the area of fibrotic lung disease and pulmonary hypertension. 


\section{References}

1 Galie N, Humbert M, Vachiery JL, et al. 2015 ESC/ERS Guidelines for the diagnosis and treatment of pulmonary hypertension. Eur Heart J 2016; 37: 67-119.

2 Kovacs G, Herve P, Barbera JA, et al. An official European Respiratory Society statement: pulmonary haemodynamics during exercise. Eur Respir J 2017; 50: 1700578.

3 Hasler ED, Muller-Mottet S, Furian M, et al. Pressure-flow during exercise catheterization predicts survival in pulmonary hypertension. Chest 2016; 150: 57-67.

4 Chaouat A, Sitbon O, Mercy M, et al. Prognostic value of exercise pulmonary haemodynamics in pulmonary arterial hypertension. Eur Respir J 2014; 44: 704-713.

5 Stamm A, Saxer S, Lichtblau M, et al. Exercise pulmonary haemodynamics predict outcomes in patients with systemic sclerosis. Eur Respir J 2016; 48: 1658-1667.

6 Degani-Costa LH, Levarge B, Digumarthy SR, et al. Pulmonary vascular response patterns during exercise in interstitial lung disease. Eur Respir J 2015; 46: 738-749.

$7 \quad$ Herve P, Lau EM, Sitbon O, et al. Criteria for diagnosis of exercise pulmonary hypertension. Eur Respir J 2015; 46: $728-737$.

8 Goh NS, Desai SR, Veeraraghavan S, et al. Interstitial lung disease in systemic sclerosis: a simple staging system. Am J Respir Crit Care Med 2008; 177: 1248-1254.

9 Cox DR. Regression models and life-tables. J R Stat Soc Series B Stat Methodol 1972; 34: 187-220.

10 Caminati A, Cassandro R, Harari S. Pulmonary hypertension in chronic interstitial lung diseases. Eur Respir Rev 2013; 22: 292-301.

11 Behr J, Ryu JH. Pulmonary hypertension in interstitial lung disease. Eur Respir J 2008; 31: 1357-1367.

12 Awano N, Inomata M, Ikushima S, et al. Histological analysis of vasculopathy associated with pulmonary hypertension in combined pulmonary fibrosis and emphysema: comparison with idiopathic pulmonary fibrosis or emphysema alone. Histopathology 2017; 70: 896-905.

13 Godinas L, Lau EM, Chemla D, et al. Diagnostic concordance of different criteria for exercise pulmonary hypertension in subjects with normal resting pulmonary artery pressure. Eur Respir J 2016; 48: $254-257$.

14 Abernethy AD, Stackhouse K, Hart S, et al. Impact of diabetes in patients with pulmonary hypertension. Pulm Circ 2015; 5: 117-123.

15 Ley B, Collard HR, King TE. Clinical course and prediction of survival in idiopathic pulmonary fibrosis. Am J Respir Crit Care Med 2011; 183: 431-440. 